\title{
Dietary Modification Guidelines for Diabetic Patients: Are Final Year Nursing Students Prepared to Offer this Health Service?
}

\author{
Nsiah-Asamoah C1*, Okantey C2 and Bamere GN3 \\ 1University of Cape Coast, Cape Coast, Ghana \\ ${ }^{2}$ School of Nursing and Midwifery, Department of Adult Health, University of Cape \\ Coast, Ghana \\ ${ }^{3}$ Department of Health Sciences Education, University of Cape Coast, Ghana
}

Research Article

Volume 2 Issue 2

Received Date: March 02, 2018

Published Date: March 16, 2018

*Corresponding author: Christiana Nsiah Asamoah, Department of Health Sciences Education, University of Cape Coast, Ghana, Tel: +233502157527; E-mail: cbuxton@ucc.edu.gh

\begin{abstract}
It has been indicated that although the responsibility of offering nutrition education lies in the hands of mainly registered dietitians and certified diabetes educators, these professionals may not be available to diabetics as often as nurses. However, a review of the literature suggests that the nutritional knowledge level of nurses in the nutritional management of chronic health conditions may be low. Therefore this study was undertaken to assess the knowledge levels of final year nursing students regarding the nutritional management of Type II diabetes mellitus. The study design was cross-sectional and it was undertaken in two randomly selected Nursing Training Institutions in Ghana. A structured pretested questionnaire was administered among final year nursing students. Although respondents had adequate knowledge about the risk factors, signs and symptoms and preventive measures of diabetes, their knowledge levels on dietary management of diabetic conditions were poor in the areas of fat intake, use of glycemic index diets, reduction of intake of refined carbohydrate foods and alcohol intake in diabetic patients. The knowledge levels of the nursing students regarding the nutritional management of diabetes were not optimal, underscoring and perhaps suggesting the need to improve upon the content areas of nutrition courses that are taught in Nursing Training Institutions. It is recommended that dietitians are included in developing the curriculum of nutrition-related courses for training nurses.
\end{abstract}

Keywords: Nursing Students; Type II Diabetes Mellitus; Dietary Management 


\section{Nursing \& Healthcare International Journal}

Abbreviations: CDEs: Certified Diabetes Educators; DM: Diabetes mellitus; DHA: Docosahexaenoic acid; EPA: Eicosapentaenoic acid; GI: Glycemic Index; MUFA: Monounsaturated Fatty Acids; NTIs: Nursing Training Institutions; RDs: Registered Dietitians; SFA: Saturated Fatty Acids; WHO: World Health Organization.

\section{Introduction}

Type 2 diabetes mellitus (T2DM) is one of the most common chronic diseases in both Western and developing countries [1]. According to the World Health Organization(WHO), the global prevalence of diabetes among adults over 18 years of age has risen from $4.7 \%$ in 1980 to $8.5 \%$ in 2014 [2]. It has been asserted that developing countries will bear the greatest brunt of the diabetes epidemic in the 21st century, with $80 \%$ of all new cases expected to appear in the developing countries like Ghana by 2025 [3]. There are also anticipations that a higher proportion of the projected numerical increase in the global prevalence of DM is to occur in developing countries [1]. There are also reports that $80 \%$ of people with diabetes live in low and middle-income countries with an estimated 522, 600 people in Sub-Saharan Africa reported to have died as a result of diabetes-related causes in 2013 [4]. Diabetes is a major cause of blindness, kidney failure, heart attacks, stroke and lower limb amputation [2].

In a review of previous studies which assessed the prevalence of diabetes in Ghana, it was indicated that the prevalence of diabetes has risen from $0.2 \%$ in the late 1950s (in a study conducted in the Volta Region) to $6.0 \%$ in 2009 (in a study undertaken in Kumasi, Ashanti Region). In Accra, a prevalence of $6.4 \%$ was recorded in a mixed community in the late 1990s and $9.1 \%$ among civil servants in 2006 [5].

Diabetes can be treated and its consequences can be prevented or delayed with diet, physical activity, medication and regular screening. Eating a healthy diet in addition to engaging in regular physical activity, maintaining a normal body weight and avoiding tobacco use are ways to prevent or delay the onset of type 2 diabetes [2]. A study which assessed the prevalence and determinants of diabetes among older adults in Ghana highlighted the need for physical activity and nutrition to be emphasized in any diabetic prevention strategy [6].

Although, the responsibility of educating diabetics on adopting and consuming healthy dietary meals lies primarily in the hands of registered dietitians (RDs) and certified diabetes educators (CDEs), these professionals may not be available to patients as often as nurses, who constitute the largest proportion of professional health worker [7].

Studies conducted in the United States and Australia revealed that $90 \%$ of nurses on a regular basis receive request for nutrition advice from patients [8]. To this end, there have been recommendations on the need for nurses to be adequately knowledgeable about nutrition therapy for diabetes patients in order to provide effective advice. However, it has been reported that, generally, nurses do not achieve appropriate nutritional education and because of insufficient knowledge and skills cannot play an effective role in patient's nutrition management [9-11]. A review of the literature suggests that the current knowledge levels of nurses on nutritional management of diabetes may be low $[9,12,13]$. Findings from these studies reported that nurses had inadequate knowledge of several aspects of nutrition management for diabetes, and they generally did poorly in relation to meal planning for diabetic patients. In an integrative review based on 25 related studies, the authors concluded that, generally, findings from the studies indicated wide-spread serious deficiencies in nurses' knowledge of diabetes and diabetes care [14].

Consequently, in studies that were conducted to assess the therapeutic nutritional knowledge of nurses it was concluded that there is an urgent need to improve the curricula of undergraduate nursing courses regarding nutrition issues and to promote postgraduate continuing educational programs concerning therapeutic nutrition $[8,15]$.

With regard to the knowledge levels of nursing students who are about completing their training, such studies is almost non-existent and no study has elaborately covered nursing students' knowledge in the nutritional management of diabetes.

The purpose of this study was to evaluate the knowledge level of final year nursing students' with regard to the nutritional management of diabetes. In addition, factors associated with nursing students' knowledge levels were also assessed. Findings from this study can provide the needed information for the revision of nutrition-related courses taught in Nursing Training Institutions. 


\section{Nursing \& Healthcare International Journal}

\section{Methods}

\section{Research Setting and Sample}

This cross-sectional study was conducted among final year nursing students who had barely two months to complete their education in two randomly selected Nursing Training Institutions (NTIs) located in one of the regions in the Northern Sector of Ghana.

All the three solely NTIs in the region were listed and two NTIs were selected for the study through the process of lottery. The target population for this study included all final year nursing students provided they gave their consent to participate in the study. Out of a total number of 210 respondents who gave their consent to participate in the study and who were given the questionnaire, 199 students completed the questionnaire representing a response rate of $94.7 \%$.

\section{Data Collection Instrument}

A questionnaire comprised of three sections, A, B and C. Section A included questions to obtain demographic data on the study participants. Section B consisted of questions which assessed respondents' knowledge levels in the risk/pre-disposing factors, signs and symptoms and prevention of diabetes. Finally, question items in Section C focused on assessing respondents' knowledge levels in the nutritional management of diabetes.

The items in Section $C$ which assessed the knowledge levels of respondents regarding the nutritional management of diabetes was developed based on the WHO nutrition guidelines for managing diabetic conditions and other published related studies that assessed the knowledge levels of health professionals in the nutritional management of diabetics $[8,15]$. The questionnaire was sent to two experts both registered dietitian to evaluate the items on the questionnaire with respect to its content validity, test format and item construction. All items were revised based on suggestions given by the dietitians and were resubmitted for further assessment. All the items were approved by the dietitians as being appropriate with good content validity. The internal consistency of the items on the questionnaire was assessed using Cronbach's alpha and a reliability coefficient of 0.72 was attained for the items. Questions that were asked assessed students' knowledge on the risk factors, signs and symptoms of diabetes and basic nutritional recommendations for diabetic patients. With regard to the questionnaire's format, some of the items were multiple choice questions while others required students to answer by either selecting either true or false. The questionnaire took approximately 30 minutes to complete.

\section{Data Collection}

The researchers administered the questionnaires in the two schools after obtaining permission from the Principal, the College management and authorities. In both schools, after explaining the purpose of the study which was also summarized on information sheets, students were allowed to ask questions or make inquiries to seek further clarification before deciding whether to volunteer to participate in the study or not. The questionnaires were given to only final year students who consented voluntarily to participate in the study by signing an inform consent form. Students who agreed to participate in the study were asked to complete the questionnaire in their lecture room and were not allowed to communicate with other class mates or take the questionnaire out of the lecture room. The students were informed by the researchers that the study was not being conducted to grade them but to obtain an idea about their nutritional knowledge in the management of diabetes for academic purposes. In addition they were told to answer questions that they could and leave out questions that they had no idea about or indicate by ticking the option "I do not know" as this was included in the answer options .

The introduction attached to each questionnaire had information assuring students anonymity and confidentiality of all data provided. The students were informed that the following steps would be taken:

1) Questionnaires administered would be anonymous and will not be used to identify any personal information.

2) Signing on the informed consent form attached to the questionnaire, completing the questionnaire in the lecture hall and not referring to any textbook or consulting a mate.

3) The questionnaire would be confidentially treated, kept safely under lock and key within the University and the electronic data files will be password protected.

4) The questionnaires would be stored for at least 5 years after publishing the data in an academic journal and subsequently shredded and discarded.

5) Anonymity would be ensured when the data is published in an academic journal

In addition, no student was required to write his or her name on the questionnaire, but the researchers assigned identity numbers to each questionnaire not to identify any 


\section{Nursing \& Healthcare International Journal}

student with a questionnaire but rather to enable data to be managed and entered in an organized manner.

\section{Data Analysis}

The data was analysed using the Statistical Package for the Social Sciences (SPSS) programme, (version 21.0). Reliability and internal consistency of the questionnaire were evaluated using Cronbach's alpha and a reliability coefficient of 0.72 was attained for the items. Also a $95 \%$ Confidence interval was used in analyzing the results from the study. Descriptive statistics were run to summarize the data collected; and the results were displayed in frequencies, percentages and means. All categorical data were expressed as frequencies and proportions.

\section{Results}

The results of the study are summarized and presented in subsequent tables. The data collected covered the following: background information of respondents, basic knowledge on diabetes, classification of diabetes, signs and symptoms of diabetes, complications of diabetes, risk factors of diabetes, prevention of diabetes, management of diabetes, knowledge on nutritional management of diabetes and nutritional recommendations for persons with diabetes.

\section{Background Information of Respondents}

The background information of the final year nursing students who participated in the study is presented in (Table 1).

\begin{tabular}{|c|c|c|}
\hline Variable & Frequency & Percentage (\%) \\
\hline \multicolumn{3}{|c|}{ Sex } \\
\hline Male & 95 & 47.7 \\
\hline Female & 104 & 52.3 \\
\hline \multicolumn{3}{|c|}{ Age } \\
\hline 20 to 23 years & 114 & 57.3 \\
\hline 24 to 27 years & 81 & 40.7 \\
\hline 28 years and above & 4 & 2 \\
\hline \multicolumn{3}{|c|}{ Do you have diabetes? } \\
\hline Yes & 4 & 2 \\
\hline No & 150 & 75.4 \\
\hline Do not know & 45 & 22.6 \\
\hline \multicolumn{3}{|c|}{ How many years/months have you had diabetes? } \\
\hline Less than 6 months & 0 & 0 \\
\hline 7 to 12 months & 1 & 25 \\
\hline 1 to 2 years & 3 & 75 \\
\hline More than 2 years & 0 & 0 \\
\hline \multicolumn{3}{|c|}{ Do you have a relative/ family member with diabetes? } \\
\hline Yes & 28 & 14.1 \\
\hline No & 139 & 69.8 \\
\hline Do not know & 32 & 16.1 \\
\hline
\end{tabular}

Table 1: Background information of respondents.

Majority $(52.3 \%)$ of the study participants were females as the nursing profession is dominated by females. Most of the respondents, 114 (57.3\%), were between the ages of 20 and 23 years. This is an indication that most of the respondents are still in their youthful age and also shows a productive human resource in the delivery of health care for the country in the near future. With regard to the diabetes condition, most $150(75.4 \%)$ of the respondents indicated that they were not diabetic, whereas $45(22.6 \%)$ did not know about their diabetic status and $4(2.0 \%)$ indicated that they were diabetics.

A summary of the findings of questions that were asked to assess the knowledge levels of the final year nursing students on the causative factors and complications associated with diabetes is presented in (Table 2). The 


\section{Nursing \& Healthcare International Journal}

questions that were asked assessed their knowledge with regard to factors associated with a high prevalence of diabetes, other health conditions that are associated with diabetes or complications of diabetes.

\begin{tabular}{|c|c|c|}
\hline Abbreviated Question & Frequency & Percentage (\%) \\
\hline \multicolumn{3}{|c|}{ What causes diabetes? } \\
\hline Low blood glucose/sugar & 8 & 4 \\
\hline High blood glucose/sugar & 191 & 96 \\
\hline \multicolumn{3}{|c|}{ How you first heard about diabetes? } \\
\hline Health facility & 19 & 9.5 \\
\hline $\mathrm{TV} /$ radio & 52 & 26.1 \\
\hline Poster/sticker & 8 & 4 \\
\hline Leaflet/ Newspaper & 12 & 6 \\
\hline Friend/relative & 8 & 4 \\
\hline School & 100 & 50.3 \\
\hline \multicolumn{3}{|c|}{ Globally, most people with diabetes are aged between... } \\
\hline Less than 20 years & 4 & 2 \\
\hline 20 to 39 years & 32 & 16.1 \\
\hline 40 to 59 years & 100 & 50.2 \\
\hline 60 years and above & 63 & 31.7 \\
\hline \multicolumn{3}{|c|}{ The high prevalence of diabetes in the country is associated with... } \\
\hline Rural dwellers & 8 & 4 \\
\hline Urban dwellers & 128 & 64.3 \\
\hline Both rural and urban dwellers & 63 & 31.7 \\
\hline \multicolumn{3}{|c|}{ Diabetes and obesity are closely related } \\
\hline Yes & 115 & 57.8 \\
\hline No & 84 & 42.2 \\
\hline \multicolumn{3}{|c|}{ Diabetes is related to hypertension } \\
\hline Yes & 68 & 34.2 \\
\hline No & 131 & 65.8 \\
\hline \multicolumn{3}{|c|}{ Chronic kidney failure and foot ulcer are some complications of diabetes } \\
\hline Yes & 119 & 59.8 \\
\hline No & 77 & 38.7 \\
\hline Do not know & 3 & 1.5 \\
\hline \multicolumn{3}{|c|}{ Group of individuals with a higher risk of developing diabetes } \\
\hline Children & 16 & 8 \\
\hline Adolescence & 12 & 6 \\
\hline Young adults & 40 & 20.1 \\
\hline Older adults & 131 & 65.9 \\
\hline
\end{tabular}

Table 2: Respondents' Knowledge on Causative Factors and Health Conditions related to Diabetes.

As many as 191 (96.0\%) of the respondents who had knowledge on what diabetes is, indicated that it was a sugar disease, mainly caused by eating too much sugar.

Participants' information on diabetes were from different sources, the most common one being from lessons taught in school, representing $50.3 \%$ of the total respondents, followed by the media (mainly television and radio) with $26.1 \%$, others, 19 (9.5\%) and 12 (6.0\%) of these respondents also heard about diabetes for the first time from the health facility and leaflets/newspaper respectively. Furthermore, more than half of the respondent's representing $50.2 \%$ indicated that most people with diabetes are aged between 40 and 59 years. Others 63(31.7\%) also indicated that the prevalence of diabetes was also common among people above 60 years.

Table 2 further depicts that majority (64.3\%) of the respondents indicated that the high prevalence of 


\section{Nursing \& Healthcare International Journal}

diabetes in Ghana is associated with urban dwellers whereas $31.7 \%$ stated that the high prevalence is associated with both rural and urban dwellers.

The students were asked whether diabetes and obesity are closely related and majority (57.8\%) of the respondents indicated that they are related. Yet, as shown in Table 2, majority (65.8\%) of the students did not know that hypertension is related to Type 2 diabetes mellitus.

With regard to respondents' knowledge on groups of individuals with a higher risk of developing diabetes, response given by the respondents is presented in Table
2. More than half $(65.9 \%)$ of the respondents indicated that diabetes is more likely to occur among older adults while some $(20.1 \%)$ indicated that diabetes is likely to occur more in younger adults.

A summary of the findings of questions that were asked to assess the knowledge levels of the final year nursing students on the risk factors, signs and symptoms, prevention and management of diabetes is shown in Table 3. The questions that were asked assessed their knowledge with regard to the risk factors, signs and symptoms, prevention and management of diabetes.

\begin{tabular}{|c|c|c|}
\hline Abbreviated Question & Frequency & Percentage (\%) \\
\hline \multicolumn{3}{|c|}{ Risk factors of diabetes } \\
\hline High intake of sugar & 193 & 96.9 \\
\hline Age greater than 40 & 113 & 56.8 \\
\hline Excessive alcohol consumption & 94 & 47.2 \\
\hline Family history of diabetes & 99 & 47.9 \\
\hline Not being physically active & 129 & 64.8 \\
\hline Smoking & 97 & 48.7 \\
\hline High intake of fat or cholesterol diet & 132 & 66.3 \\
\hline Excess weight & 144 & 72.4 \\
\hline \multicolumn{3}{|c|}{ Signs and symptoms of diabetes } \\
\hline Excessive thirst & 139 & 69.8 \\
\hline Tiredness and weakness & 103 & 52.8 \\
\hline Frequent urination & 134 & 67.3 \\
\hline Unexplained Weight loss & 123 & 61.8 \\
\hline Headache & 47 & 23.6 \\
\hline Wounds that heal very slowly & 166 & 83.4 \\
\hline Blurred vision & 77 & 38.7 \\
\hline \multicolumn{3}{|c|}{ Can diabetes be prevented? } \\
\hline Yes & 175 & 87.9 \\
\hline No & 24 & 12.1 \\
\hline \multicolumn{3}{|c|}{ How diabetes can be prevented } \\
\hline Healthy diet & 163 & 93.1 \\
\hline Regular exercise & 125 & 71.4 \\
\hline Taking herbs & 35 & 20 \\
\hline Weight reduction & 101 & 57.7 \\
\hline Not smoking & 91 & 52 \\
\hline Moderate alcohol consumption & 97 & 55.4 \\
\hline Do not know & 4 & 2 \\
\hline \multicolumn{3}{|c|}{ Ultimate goal of diabetes management } \\
\hline Maintain blood glucose levels within the normal range & 96 & 48.2 \\
\hline Deal with burden on relatives & 4 & 2.1 \\
\hline Prevent complications & 80 & 40.2 \\
\hline Avoid psychological stress & 19 & 9.5 \\
\hline
\end{tabular}

Table 3: Respondents' Knowledge on Risk factors, Signs and Symptoms, Prevention and Management of Diabetes. 


\section{Nursing \& Healthcare International Journal}

Almost all (96.9\%) of the respondents indicated that high intake of sugar is a potential risk factor of diabetes. In addition, more than $50 \%$ of the respondents also indicated that age greater than 40 years, not being physically active, high intake of fat or cholesterol diet and excessive weight gain are other risk factors of diabetes. However, less than $50 \%$ of the respondents knew that excessive intake of alcohol, family history of diabetes and smoking are risk factors of diabetes.

Regarding respondents' knowledge on the sign and symptoms of diabetes, the highest proportion (83.4\%) indicated that wounds that heal very slowly are one of the symptoms of diabetes. More than $50 \%$ but less than $70 \%$ of the respondents knew that other signs and symptoms of diabetes are excessive thirst, tiredness and weakness, frequent urination and unexplained weight loss. However, less than $40 \%$ knew that headache $(23.6 \%)$ and blurred vision (38.7\%) are other signs and symptoms of diabetes.

With regard to whether diabetes can be prevented, a higher proportion $(87.9 \%)$ of the respondents indicated that it can be prevented. Most (93.1\%) of the study participants indicated that diabetes can be prevented by eating a healthy diet. Again, 71.4\%, 57.7\%, 55.4\% and $52.0 \%$ knew that engaging in regular exercise, weight reduction, moderate alcohol consumption and not smoking respectively are all other preventive measures of diabetes.

However, some of the respondents (20\%) indicated that diabetes can be prevented by taking in herbs such as garlic.

With respect to the ultimate or major goal of any diabetes management programme or therapy, most $(48.2 \%)$ of the respondents indicated that it is ensuring that blood glucose levels falls within the normal range. In addition, others (40.2\%) indicated that preventing complications should be the ultimate goal of any diabetes management programme or therapy.

The main focus of this study was to assess final year students' knowledge levels on nutritional management of diabetic condition. Responses given by students are presented in Table 4.

\begin{tabular}{|c|c|c|}
\hline \multirow{2}{*}{ Abbreviated Question } & \multicolumn{2}{|r|}{ YES } \\
\hline & Frequency & Percentage (\%) \\
\hline Diabetics should not exclude any nutrient from their diet & 96 & 48.2 \\
\hline Monitoring carbohydrate intake is a key strategy in achieving glycemic control & 127 & 63.8 \\
\hline The total amount of carbohydrates is more important than the type of carbohydrate & 91 & 45.7 \\
\hline Increase intake of long-chain omega-3 fatty acids from fatty fish is recommended & 55 & 27.6 \\
\hline Reduce or avoid intake red and processed meats & 102 & 51.3 \\
\hline Adopt eating meals rich in Monounsaturated and Polyunsaturated fatty acids & 90 & 45.2 \\
\hline Animal fat should be restricted for diabetics with dyslipidemia & 144 & 72.4 \\
\hline Saturated fat should be reduced for diabetics & 164 & 82.4 \\
\hline$<7 \%$ of the total caloric intake of diabetics can come from saturated fat & 50 & 25.1 \\
\hline Diabetics who wish to drink alcohol, should do so with meals & 4 & 2 \\
\hline
\end{tabular}

Table 4: Respondents' Knowledge on nutrition therapy to manage diabetes.

Respondents' were required to indicate whether diabetics should not exclude any major nutrient from their diet, of which majority (51.8\%) responded in the negative as shown in Table 4. As shown in Table 4, majority $(63.8 \%)$ of the respondents knew that monitoring carbohydrate intake is a key strategy in achieving glycemic control for diabetics. Although evidence is inconclusive for the ideal amount of carbohydrate intake for people with diabetes, the proportion of carbohydrate in the meal and available insulin remains a key strategy in achieving glycemic control after eating and should be taken into consideration when developing an eating plan [18]. Less than half $(45.7 \%)$ were able to indicate that for diabetics, the total amount of carbohydrates is more important than the type of carbohydrate that is consumed. 


\section{Nursing \& Healthcare International Journal}

A higher proportion (72.4\%) of the respondents did not know that diabetics should be advised to increase their intake of long-chain omega-3 fatty acids, such as Eicosapentaenoic acid (EPA) and docosahexaenoicacid (DHA), mainly from fatty fish. A little over half (51.3\%) of the respondents indicated that they agreed that diabetics should reduce or avoid the intake of red and processed meats. Less than half $(45.2 \%)$ of the respondents were able to indicate that diabetics should be encouraged to consume meals that are rich in monounsaturated and polyunsaturated fatty acids.
Majority, $72.4 \%$ and $82.4 \%$ were able to indicate that animal fat should be restricted for diabetics with dyslipidemia and saturated fat should be restricted for diabetics respectively. However, only $25.1 \%$ of the respondents knew that $<7 \%$ of the total caloric intake of diabetics can come from saturated fat.

Almost all (98.0\%) of the respondents did not know that diabetics who choose to drink alcohol in moderate quantities should consume their alcoholic beverage with meals and not consume the alcohol alone based on evidence of its negative effects in literature.

\begin{tabular}{|c|c|c|}
\hline \multirow{2}{*}{ Abbreviated Question } & \multicolumn{2}{|r|}{ YES } \\
\hline & Frequency & Percentage (\%) \\
\hline Reduce intake of trans fat & 71 & 35.7 \\
\hline $\begin{array}{l}\text { Time meals appropriately, keeping mealtimes constant daily, if on a premixed or fixed } \\
\text { insulin plan }\end{array}$ & 54 & 27.1 \\
\hline $\begin{array}{l}\text { Eat three (3) main meals per day plus snacking on mainly fruits to balance the peak } \\
\text { activity of insulin }\end{array}$ & 58 & 29.1 \\
\hline Encourage higher fibre carbohydrate foods & 106 & 53.3 \\
\hline Recommended to consume low-glycemic index (GI) diet & 55 & 27.6 \\
\hline Decrease consumption of refined carbohydrates such as white rice and white bread & 89 & 44.7 \\
\hline
\end{tabular}

Table 5: Respondents' knowledge on Nutritional recommendations for Diabetics.

The study participants were asked some questions on nutritional recommendations that they will give to diabetic patients. Responses given to these questions are presented in Table 5. A higher proportion (64.3\%) of the study participants indicated that they will not recommend a reduction in the intake of trans fats for diabetic patients. Another finding that raises concern is that more than $70 \%$ of the nursing students did not know that when diabetic patients are on a premixed or fixed insulin plan, meals have to be consumed at similar times every day. Most (70.9\%) of the respondents did not correctly indicate that it is advisable for diabetics to eat three (3) main meals per day in addition to snacking on mainly fruits between meals to balance the peak activity of insulin. A little over half $(53.3 \%)$ of the respondents indicated that diabetics are to be advised to consume more of high fibre foods. Majority (72.4\%) of the study participants indicated that they will not advise diabetics to consume more of low-glycemic index (GI) diets.

As shown in Table 5, majority (55.3\%) of the study participants did not know that diabetics are to be cautioned to reduce or avoid the consumption of refined carbohydrates food items such as white bread and white rice.

\section{Discussion}

The results of this study showed that more than half of the respondents had information on diabetes from lesson taught in school and therefore suggests that most nursing students are likely to never hear of diabetes until they gain admission into the Nursing Training College and also implies that training received by students from Health Training Institutions plays a key role in introducing them to chronic health conditions and preparing them to support patients afflicted by these conditions.

More than $50 \%$ of the respondents knew that engaging in regular exercise, weight reduction, moderate alcohol consumption and not smoking are all other preventive measures of diabetes. These findings suggest that most of the students have an idea about strategies that can be 


\section{Nursing \& Healthcare International Journal}

adopted to prevent diabetes or lower its likelihood of occurring.

However some $(20 \%)$ of the respondents indicated that diabetes can be prevented by taking in herbs such as garlic. This finding call for some needed attention at correcting these wrong notions that some students have on the use of herbs to prevent or treat diabetes. The most recent American Diabetic Association position statement concludes that after a review of the evidence available on the role that herbs play in the prevention, treatment or management of diabetes, there is not enough clear data to substantiate recommending the use of herbs. The paper goes on to say that the use of any herbal supplements, which are not regulated and vary in content, may provide more risk than benefit, in that, herbs may interact with other medications that are taken to control diabetes [10].

With regard to the ultimate goal of any diabetes management programme, less than $50 \%$ of the respondents indicated that it is to ensure that blood glucose levels falls within the normal range.

However, as indicated in literature, the major goal of diabetes treatment and management is to maintain normal or near normal blood glucose levels in affected individuals $[10,16]$. The findings of this study suggest that most of the students do not know the ultimate goal of diabetes treatment and management plan and this may negatively influence their nursing care practices.

With regard to whether the total amount of carbohydrates is more important than the type of carbohydrate for diabetics, less than half (45.7\%) were able to provide the right answer. In a related study which was conducted among professional practicing nurses in a Ghana, over $50 \%$ of the nurses did not know that the total amount of carbohydrates consumed per meal is generally more important than the type of carbohydrate consumed [9].

The finding that more than $70 \%$ of the respondents did not know that diabetics should be advised to increase their intake of long-chain omega-3 fatty acids, such as EPA and DHA, mainly from fatty fish is of great concern and worrying and suggests that nursing trainees lack some knowledge about appropriate dietary practices that must be recommended to diabetics to help them manage their health condition. Perhaps, these students might not have received adequate education on the role of fatty acids in preventing and managing diabetic conditions.
Only $51.3 \%$ of the respondents agreed that diabetics should reduce or avoid the intake of red and processed meat products and this finding deserves much concern as the current knowledge of these final year nursing students may inform the kind of nutritional advice given to diabetic clients who access health facilities to manage their health condition. There is ample evidence in literature that highlights that a high total intake of animal protein but not vegetable protein intake is associated with diabetes risk $[17,18]$.

This study revealed that more than $50 \%$ of the nursing students did not know that diabetics should be encouraged to consume meals that are rich in monounsaturated and polyunsaturated fatty acids. This warrants great concern regarding the knowledge levels of these final year nursing trainees regarding the different types of fatty acids and how they either positively or negatively influence the management of diabetic conditions. It has been shown that diets rich in monounsaturated fatty acids (MUFA) may benefit glycemic control and cardiovascular risk factors and can therefore be recommended as an effective alternative to a lower-fat, higher-carbohydrate eating pattern among diabetics [10]. There is some evidence that replacing saturated fatty acids (SFA) with MUFA improves insulin responsiveness in insulin-resistant and type 2 diabetic subjects [19].

Although majority of the study participants knew that intake of animal fat and saturated fat should be restricted in diabetics, just approximately one fourth $(25.1 \%)$ of the respondents were able to indicate that $<7 \%$ of the total caloric intake of diabetics can come from saturated fat. Indeed, the 2013 Standards of Medical Care for Diabetes from the American Diabetes Association recommends that saturated fat intake should be less than or equal to $7 \%$ of total calories and encourages replacing high trans-fat partially hydrogenated vegetable oils and animal fats with healthier oils and foods higher in unsaturated fats such as monounsaturated and polyunsaturated [10].

It is worrying that findings of this study suggest that almost all the nursing students are not well- informed about the need to caution alcoholics who are also diabetics that apart from reducing their alcohol intakes, they should always ensure that whenever they have the urge to drink, they should drink the alcoholic beverage with a meal and not take the drink alone on an empty stomach. It has been recommended that diabetics who want to take in alcoholic beverages should drink in moderation and consume alcohol with meals in order to 


\section{Nursing \& Healthcare International Journal}

decrease the risk of hypoglycemia, which can particularly occur in the fasting state [10]. In addition, high alcohol consumption reduces insulin sensitivity and decreases pancreatic $\beta$-cells survival, thereby significantly reducing insulin secretion [20].

Majority of the respondents indicated that they will not recommend a reduction of the intake of trans fats among diabetics. The findings suggest that maybe nursing students do not have adequate knowledge on what trans fatty acids are and its negative health effects on the general population including diabetics. The American Heart Association, and American Diabetes Association currently recommends limiting the amount of dietary saturated and trans fat intake. This is because reducing the intake of trans fat also lowers Low Density Lipoproteins cholesterol and increases High Density Lipoprotein cholesterol, therefore intake of trans fat should be minimized by diabetics [10].

More than $70 \%$ of the nursing students did not know that when diabetic patients are on a premixed or fixed insulin plan, meals have to be consumed at similar times every day as highlighted in literature [21]. This finding warrants immediate attention since nurses are expected to be knowledgeable about how and when medications should be taken in relation to the intake of food in order to appropriately advise and caution patients.

The fact that just a little over half (53.3\%) of the respondents indicated that they will advise diabetics to consume more high fibre foods raises concerns particularly because of the significant role played by dietary fibre foods in diabetics. People with diabetes are advised to consume at least the amount of fibre and whole grains recommended for the general public [11]. A higher proportion of the respondents $(72.4 \%)$ did not agree that it is recommended to advise diabetics to consume more of low-glycemic index (GI) diets. Based on some studies, it has specifically been recommended to use low-glycemic index diets to improve glycemic control [11,22].

A higher proportion of the respondents (55.3\%) indicated that they will not caution diabetics to reduce or avoid the intake of refined carbohydrates food items such as white bread and white rice. This finding is worrying because of the ample evidence summarized in two metaanalysis, all of which eventually concluded that a high consumption of white rice is associated with a significantly increased risk of Type 2 diabetes Mellitus $[18,23]$.

\section{Conclusion}

Although this study showed that final year nursing students were knowledgeable in the risk factors and signs and symptoms of T2DM, their knowledge in the aspects of nutritional therapy for the prevention and management of T2DM was sub-optimal. Findings from this study demonstrate and reiterate the urgent need to promote continuing education programmes in the area of therapeutic nutrition/dietary modification for diabetic patients. With regard to possible methods of further education, a recommendation is the need to update and strengthen the content of the nutrition curriculum of diploma and degree-awarding nursing programmes in collaboration with experts in nutrition such as dieticians. It is also worth considering conducting regular refresher workshops for nurse tutors/educators who teach nutrition-related courses to help them update their knowledge in current trends of managing health conditions like T2DM. In addition, it is worthwhile to advocate for the development of a dietary modification resource toolkit for chronic conditions like T2DM which can be adopted for use by all nursing training institutions. Another helpful suggestion will be the need to consider giving nursing students the opportunity to apply their theoretical knowledge during practical clinical field experience in hospital settings while being mentored by dieticians as part of their training.

\section{Conflict of Interests}

The authors declare that there is no conflict of interest regarding the publication of this paper.

\section{Funding}

This research received no specific grant from any funding agency in the public, commercial, or not-for-profit sectors.

\section{Authors' contributions}

Christiana Nsiah-Asamoah conceived the idea of the study, contributed to the study design, assisted with developing the survey instruments, wrote the draft manuscript and contributed to final version of manuscript. Christiana Okantey contributed to the study design, assisted with developing the survey instruments, and contributed to final version of manuscript. Guido Nyellayeng Bamere contributed to the study design, assisted with developing the survey instruments, 


\section{Nursing \& Healthcare International Journal}

collected and analyzed the data and contributed to the final version of the manuscript.

All the authors read and approved the final manuscript.

\section{Acknowledgements}

The authors would like to acknowledge the effort of all the final year nursing students for their participation in the survey and tutors for their great help and support with the data collection.

\section{References}

1. Cook Huynh M, Ansong D, Steckelberg RC, Boakye I, Seligman K, et al. (2012) Prevalence of hypertension and diabetes mellitus in adults from a rural community in Ghana. Ethn Dis 22(3): 347-352.

2. World Health Organization (2012) Global report on diabetes.

3. Mumu SJ, Saleh F, Ara F, Haque MR, Ali L (2014) Awareness regarding risk factors of type 2 diabetes among individuals attending a tertiary-care hospital in Bangladesh: a cross-sectional study. BMC Res Notes 7: 599 .

4. Aguiree F, Brown A, Cho NH, Dahlquist G, Dunning S, et al. (2013) International Diabetes Federation (IDF). Diabetes Atlas.

5. De Graft Aikins A, Owusu Dabo E, Agyemang C (2014) Diabetes in Ghana: A Review of Research on Prevalence, Experiences and Healthcare. Chronic Non-communicable Diseases in Ghana. Multidisciplinary Perspectives.

6. Gatimu SM, Milimo BW, Sebastian MS (2016) Prevalence and determinants of diabetes among older adults in Ghana. BMC Public Health 16(1): 1174.

7. Bureau of Labor Statistics (2015) Registered nurses have highest employment in healthcare occupations; anesthesiologists earn the most. Postal Square Building, 2 Massachusetts Avenue, NE Washington, DC, United States of America.

8. Carney TA (2010) Knowledge, comfort level and the perceived role of nurses in promoting nutritional management of diabetes, (Doctoral dissertation, Drexel University).
9. Mogre V, Ansah AG, Marfo DN, Garti AH (2015) Assessing nurses' knowledge levels in the nutritional management of diabetes. IJANS 3: 40-43.

10. Evert AB, Boucher JL, Cypress M, Dunbar SA, Franz MJ, et al. (2013) Diabetes Association. Nutrition therapy recommendations for the management of adults with diabetes. Diabetes Care 36(11): 38213842.

11. Abdollahi M, Houshiarrad A, Abtahi M, Pouraram M, Esmaeli H, et al. (2013) The nutrition knowledge level of physicians, nurses and nutritionists in some educational hospitals. J Paramed Sci 4.

12. Hollis M, Glaister K, Anne Lapsley J (2014) Do practice nurses have the knowledge to provide diabetes selfmanagement education? Contemp Nurse 46(2): 234241.

13. Oyetunde MO, Famakinwa TT (2014) Nurses' knowledge of contents of diabetes patient education in Ondo-state, Nigeria. J Nurs Educ Pract 4(4): 91.

14. Alotaibi A, Al Ganmi A, Al Ganmi, Gholizadeh L, Perry L (2016) Diabetes knowledge of nurses in different countries: An integrative review. Nurse Educ Today 9: 32-49.

15. Al Shwaiyat NM, Sinjillawi AB, Al Rethaiaa AS, Fahmy AEA, Aqel RM, et al. (2013) Assessment of therapeutic nutritional knowledge of Jordanian nurses. International Journal of Nutrition and Food Sciences 2(3): 142-148.

16. Shrivastava SR, Shrivastava PS, Ramasamy J (2012) Role of self-care in management of diabetes mellitus. J Diabetes Metab Disord 12: 14.

17. Feskens EJ, Sluik D, vanWoudenbergh GJ (2013) Meat consumption, diabetes, and its complications. CurrDiabRep 13(2): 298-306.

18. Hu EA, Pan A, Malik V, Sun Q (2012) White rice consumption and risk of type 2 diabetes: metaanalysis and systematic review. Bmj 344: e1454.

19. Odegaard AO (2013) Dietary fatty acids in the etiology of type 2 diabetes. In Nutrition and Type 2 Diabetes: Etiology and Prevention. CRC Press, pp: 6588. 


\section{Nursing \& Healthcare International Journal}

20. Yoo MG, Kim HJ, Jang HB, Lee HJ, Park SI (2016) The Association between Alcohol Consumption and $\beta$-Cell Function and Insulin Sensitivity in Korean Population. Int J Environ Res Public Health 13(11): 1133.

21. Bendich A, Deckelbaum RJ (2016) Preventive nutrition: The comprehensive guide for health professionals. Springer.

22. Jenkins DJ, Kendall CW, Augustin LS, Mitchell S, Sahye-Pudaruth S, et al. (2012) Effect of legumes as part of a low glycemic index diet on glycemic control and cardiovascular risk factors in type 2 diabetes mellitus: a randomized controlled trial. Arch Intern Med 172(21): 1653-1660.

23. Aune D, Norat T, Romundstad P, Vatten LJ (2013) Whole grain and refined grain consumption and the risk of type 2 diabetes: a systematic review and doseresponse meta-analysis of cohort studies. Eur. J. Epidemiol 28(11): 845-858. 